\title{
Clinical features of multidrug-resistant organism infections in early postoperative solid organ transplantation in a single center
}

\author{
Min Jin", Lijuan Zeng", Wanyi Zhang, Xuanying Deng, Jiali Li, Weiting Zhang \\ Organ Transplantation Center, Panyu Campus, the Second Affiliated Hospital of Guangzhou Medical University, Guangzhou, China \\ Contributions: (I) Conception and design: M Jin, L Zeng; (II) Administrative support: W Zhang; (III) Provision of study materials or patients: W \\ Zhang; (IV) Collection and assembly of data: X Deng; (V) Data analysis and interpretation: J Li; (VI) Manuscript writing: All authors; (VII) Final \\ approval of manuscript: All authors. \\ "These authors contributed equally to this work. \\ Correspondence to: Weiting Zhang. Organ Transplantation Center, Panyu Campus, the Second Affiliated Hospital of Guangzhou Medical University, \\ Guangzhou 510260, China. Email: gzzhangwt@126.com.
}

\begin{abstract}
Background: The increasing occurrence of multidrug-resistant organisms (MDROs) infections has posed major challenge to solid organ transplantation (SOT). For SOT recipients, high-dose immunosuppressants and broad-spectrum antibiotics can markedly increase the risk of early postoperative MDRO infections and thus have adverse effects on the outcomes of SOT. Here, we analyzed the incidence and clinical features of early MDRO infections after SOT, in an attempt to provide new evidence for the control and treatment of early MDROs.
\end{abstract}

Methods: The clinical data of 133 patients with MDRO infections after SOT in our department from 2017 to 2020 were retrospectively collected, and clinical features including incidence, etiologies, infection sites, and complications, were analyzed.

Results: The incidence of MDRO infections after SOT was 9.9\%. Simultaneous liver and kidney transplantation patients had the highest incidence of MDRO infections, followed by the recipients of liver transplantation and simultaneous pancreas-kidney transplantation; patients undergoing renal transplantation had the lowest incidence of MDRO infections. The most common pathogen was extended spectrum betalactamase (ESBL)-producing organisms $(\mathrm{n}=88,66.2 \%)$, the most common infection site was the urinary system $(n=58,43.6 \%)$, and the main postoperative complications were urinary tract infections $(n=44,33.1 \%)$ and lung infections $(\mathrm{n}=41,30.8 \%)$. MDRO infections were cured in most cases.

Conclusions: A sound knowledge of the clinical features of MDRO infection after SOT is important for the successful prevention and treatment of these infections.

Keywords: Solid organ transplantation (SOT); multidrug-resistant organism (MDRO); infection; clinical features

Submitted Jan 20, 2021. Accepted for publication Apr 24, 2021.

doi: $10.21037 /$ apm-21-777

View this article at: http://dx.doi.org/10.21037/apm-21-777

\section{Introduction}

Solid organ transplantation (SOT) is the preferred treatment option for patients with end-stage organ disease. The number of SOT surgeries has been steadily rising in recent years, and there has been a marked increase in the median survival rates of recipients and grafts $(1,2)$. However, the increasing incidence of multidrug-resistant organism
(MDRO) infections has posed a major challenge to SOT. For SOT recipients, high-dose immunosuppressants and broad-spectrum antibiotics can markedly increase the risk of early postoperative MDRO infections and thus exert adverse effects on the outcomes of SOT.

Perioperative and postoperative infections in SOT recipients are mainly nosocomially acquired and are mostly caused by Gram-negative bacilli (3). In 2019, the Chinese 
Antimicrobial Resistance Surveillance System (CARSS) reported the total number of bacterial strains to be 3,528,471, of which Gram-positive bacteria accounted for $29.6 \%$ and Gram-negative bacteria $70.4 \%$. The strains were mainly cultured from sputum $(1,462,853$ strains, $41.5 \%)$, urine $(673,824$ strains, $19.1 \%)$ and blood $(32,002$ strains, $9.1 \%)$. The top 5 Gram-positive bacteria isolated were Staphylococcus aureus, Streptococcus pneumoniae, Enterococcus Faecium, Staphylococcus epidermidis, and Enterococcus faecalis, and the top 5 Gram-negative bacteria isolated were Escherichia coli, Klebsiella pneumoniae, Pseudomonas aeruginosa, Acinetobacter baumannii, and Haemophilus influenzae. Due to the differences in environment, climate, ecology, and population, the sites of early postoperative MDRO infections and the distribution of pathogenic bacteria after SOT vary somewhat across different regions (4).

Though there have been many reports in the literature about multi-drug resistant bacteria infections in organ transplant recipients $(1,2,4)$. For empirical treatment of suspected bacterial infections in SOT patients, the selection of antimicrobial agents should be based on local epidemiological data and on the patient's history of colonization or infection with multidrug-resistant organism (5). At present, there are limited reports on the clinical features of multi-resistant infections after SOT in South China. As a result, there is insufficient clinical evidence to support the outcome of the prevention and treatment of multidrug-resistant infections after SOT in South China. Furthermore, this study compared the characteristics of multi-resistant infections between different organ transplants, providing guidance for the prevention and treatment of multi-drug resistant bacteria infections after different types of transplants.

Here, we present an analysis of the incidence and clinical features (including pathogens, infection sites, postoperative complications, and prognosis) of early MDRO infections after SOT, in an attempt to provide new evidence for the control and treatment of early MDRO infections. We present the following article in accordance with the STROBE reporting checklist (available at http://dx.doi. org/10.21037/apm-21-777).

\section{Methods}

\section{Study design}

A retrospectively analysis.

\section{Setting}

A total of 133 patients with MDRO infection within 3 months after SOT admitted to the Second Affiliated Hospital of Guangzhou Medical University from January 1, 2017 to September 1, 2020 were enrolled by using cluster sampling. This study was conformed to the provisions of the Declaration of Helsinki (as revised in 2013) and was approved by the Clinical Research and Application Ethics Committee of the Second Affiliated Hospital of Guangzhou Medical University (No. 2018-QCC-ks-01). Written informed consent was obtained from the patient.

\section{Participants}

\section{Inclusion criteria}

The inclusion criteria for participants were the following: (I) aged 18 years or above; (II) receiving organ transplantation for the first time; (III) without any infection or bacterial colonization before surgery; (IV) with complete medical records; and (V) survival with 24 hours of surgery.

\section{Exclusion criteria}

The exclusion criteria for participants were the following: (I) long-term immunosuppressive therapy prior to surgery; and (II) transfer to other hospitals during treatment and/or lost to follow-up during the study period.

The clinical diagnoses of MDRO infections were based on the Diagnostic Criteria for Hospital Infections, as released by the Ministry of Health of the People's Republic of China in 2001. More specifically, the diagnostic criteria included the same bacterial strain being identified on more than 2 consecutive occasions or at more than 2 sites, and resistance to any combination of 3 or more of the 3 drug classes (carbapenems, cephalosporins, $\beta$-lactam and $\beta$-lactamase inhibitor combinations, fluoroquinolones, and aminoglycosides), then multidrug-resistant bacterial infection after Simultaneous pancreatic and kidney transplantation is diagnosed.

\section{Sduty methods}

\section{Immunosuppressive regimen}

The immunosuppressive regimen consisted of the following: a triple immunosuppressive scheme that included tacrolimus (FK506) or cyclosporine A (CsA) + mycophenolate mofetil (MMF) or azathioprine (Aza) + prednisone (Pred). 
Table 1 Incidences of multidrug-resistant organism (MDRO) infections in patients after different organ transplantation procedures

\begin{tabular}{lcccc}
\hline $\mathrm{n}$ & $\begin{array}{c}\text { Kidney } \\
\text { transplantation }\end{array}$ & $\begin{array}{c}\text { Simultaneous pancreas-kidney } \\
\text { transplantation }\end{array}$ & $\begin{array}{c}\text { Liver } \\
\text { transplantation }\end{array}$ & $\begin{array}{c}\text { Simultaneous liver-kidney } \\
\text { transplantation }\end{array}$ \\
\hline Number of patients with MDRO infections & 98 & 33 & 1 & 1 \\
Total cases & 1,172 & 166 & 4 & 1 \\
Incidence & $8.4 \%$ & $19.9 \%$ & $25 \%$ & $100 \%$ \\
\hline
\end{tabular}

\section{Main measures}

Based on the findings of literature review and our own experiences, the following items were analyzed in our current study: gender, age, primary disease, preoperative antimicrobial therapy, postoperative ICU stay, duration of postoperative intravenous catheterization, duration of postoperative urinary catheterization, postoperative immunosuppression regimen, major postoperative complications, and length of hospital stay. A clinical data collection form was established, and data were collected by reviewing the inpatient medical records and medical examination reports, and by follow-up visits.

\section{Statistical analysis}

Statistical analysis was performed using the SPSS 20.0 software package (IBM Corp. USA). Measurement data are expressed as mean \pm standard deviations, and count data are expressed in case numbers and percentages.

\section{Results}

\section{General information}

A total of 133 SOT recipients with early postoperative infection with MDRs were included in this study. Among them there were 92 allogeneic kidney transplantation patients (69.2\%), 6 living-related kidney transplantation patients (4.5\%), 33 simultaneous pancreas and kidney transplantation patients (24.8\%), 1 liver transplantation patient $(0.8 \%)$, and 1 Simultaneous liver and kidney transplantation $(0.8 \%)$. There were 66 males $(49.6 \%)$ and 67 females $(50.4 \%)$ aged $44.23 \pm 14.37$ years; 65 patients $(48.9 \%)$ had been hospitalized before surgery and 68 cases $(51.1 \%)$ had not. Preoperative antibiotics were used in 15 cases $(11.3 \%)$ and were not used in 118 cases $(88.7 \%)$. The preoperative primary diseases were chronic glomerulonephritis in 60 cases $(45.1 \%)$, diabetic nephropathy in 38 cases (28.6\%), immunoglobin
(IgA) nephropathy in 8 cases (6\%), lupus nephritis in 3 cases $(2.3 \%)$, and others in 24 cases $(18 \%)$. The duration of postoperative intravenous catheterization was $9.09 \pm 8.77$ days, the duration of indwelling urinary catheterization was $6.71 \pm 3.28$ days, the duration of postoperative ICU stay was $0.54 \pm 2.65$ days, and the duration of hospitalization was $29.17 \pm 18.74$ days. For donors, the length of ICU stay was $5.02 \pm 13.266$ days and the intubation time was $4.85 \pm 13.20$ days.

\section{Incidences of MDRO infections in patients after different organ transplantation procedures}

From January 1, 2017, to September 1, 2020, the total number of kidney transplantations, Simultaneous pancreas and kidney transplantations, liver transplantations, and Simultaneous liver and kidney transplantations performed at our center was $1,172,166,4$, and 1 , respectively. The incidences of MDRO infections in patients after different organ transplantation procedures are summarized in Table 1.

\section{Clinical features of MDRO infections in patients after organ transplantation procedures}

The clinical features including pathogens, infection sites, complications, and outcomes of MDRO infections in the early stage after different organ transplantation are shown in Table 2.

\section{Discussion}

Infections occurring early after SOT are usually surgery related, with pathogens mostly originating from the inhospital environment or carried by the donor and/or recipient. The risk of MDRO infection is particularly high in organ donors or recipients after prolonged ICU stay, where they typically receive a series of interventions (including invasive procedures). SOT recipients are often required to take long-term immunosuppressive drugs, 
Table 2 Clinical features of multidrug-resistant organism (MDRO) infections in patients after organ transplantation

\begin{tabular}{|c|c|c|}
\hline Item & Classification & $\mathrm{n}(\%)$ \\
\hline \multirow{7}{*}{ Pathogen } & Vancomycin-resistant enterococci (VRE) & $2(1.5)$ \\
\hline & Extended spectrum beta-lactamase (ESBL)-producing organisms & $88(66.2)$ \\
\hline & Carbapenem-resistant Acinetobacter baumannii (CR-AB) & $13(9.8)$ \\
\hline & Carbapenem-resistant enterobacteriaceae (CRE) & $12(9.0)$ \\
\hline & Multidrug-resistant Mycobacterium tuberculosis & 0 \\
\hline & Carbapenem-resistant Pseudomonas aeruginosa (CR-PA) & $11(8.3)$ \\
\hline & Multidrug-resistant Escherichia coli & 0 \\
\hline \multirow[t]{4}{*}{ Infection site } & Urinary tract & $58(43.6)$ \\
\hline & Lungs & $39(29.3)$ \\
\hline & Wound & $5(3.8)$ \\
\hline & Others & $44(33.1)$ \\
\hline \multirow[t]{4}{*}{ Postoperative complications } & Urinary tract infection & $44(33.1)$ \\
\hline & Pulmonary infection & $41(30.8)$ \\
\hline & Acute graft rejection & $31(23.3)$ \\
\hline & DGF & $1(0.8)$ \\
\hline \multirow{3}{*}{ Outcomes } & With severe but reversible complications & $1(0.8)$ \\
\hline & With severe and irreversible complications & $0(0)$ \\
\hline & Death & $4(3.0)$ \\
\hline
\end{tabular}

DGF, delayed graft function.

which leads to secondary immune dysfunction, resulting in a significantly increased incidence of postoperative infections. The combinations of multiple antibiotics can promote the development of multidrug-resistant bacteria while reducing the patient's risk of infection. Advanced age, higher BMI (6), underlying disease, prolonged preoperative hospitalization, and prolonged indwelling catheterization also increase the incidence of MDRO infections. In addition, surgery-related risk factors for MDRO infections include contamination of preservation fluid, prolonged surgery, high intraoperative bleeding, retention of a foreign object, poor graft perfusion, anastomotic or intestinal fistula, inappropriate antibiotic surgical prophylaxis, and environmental factors in the operating room (3).

\section{Differences in the incidences of MDRO infections in patients after different organ transplantation procedures}

A total of 1,343 SOT procedures were performed in our center between 2017 and 2020, from which 133 
patients suffered from MDRO infections within 3 months after surgery, yielding an MDRO infection rate of $9.9 \%$, which is basically consistent with the incidences reported in literature $(7,8)$. The incidence of MDRO infection was highest after Simultaneous liver and kidney transplantation (1 simultaneous liver and kidney transplant recipient developed MDRO infection within 3 months after surgery, yielding an incidence of $100 \%$ ), followed by liver transplantation (4 liver transplant recipients developed MDRO infections within 3 months after surgery, yielding an incidence of $25 \%$ ) and Simultaneous pancreas and kidney transplantation [with an incidence of $19.9 \%(n=166)$ within 3 months after surgery]. The incidence $(8.4 \%)$ of MDRO infection was lowest among the 1,178 renal transplantation recipients. Although the small number of patients undergoing Simultaneous liver and kidney transplantation or liver transplantation in our analysis might have introduced a large sampling bias, infection is an undoubtedly frequent and lifethreatening complication after liver transplantation $(9,10)$. Zhong et al. found the rates of infection after liver transplantation ranged between $41 \%$ and $56 \%$, and that the vast majority of the pathogens were bacteria, accounting for two-thirds of all infections (11). Donor-derived bacterial infections are an important cause of MDRO infection after liver transplantation; the common pathogens include Pseudomonas aeruginosa, Klebsiella pneumoniae, and Enterobacter aerogenes (12-16), and the routes of infection include pulmonary infections, bloodstream infections (BSIs), and urinary tract infections $(17,18)$. Liver transplantation-induced stress response can cause swelling and mucosal damage in the gastrointestinal tract, leading to intestinal dysbiosis and translocation of gut flora, which can result in a high rate of intra-abdominal bacterial infections in the early postoperative period. Meanwhile, the use of long-term abdominal drains in liver transplant recipients increases the chance of abdominal infection, which in turn increases the risk of BSI. Compared to kidney transplantation alone, Simultaneous liver and kidney transplantation, liver transplantation, and Simultaneous pancreas and kidney transplantation last longer and are more challenging, with a correspondingly higher probability of intraoperative infections. The most common site of infection in kidney transplantation is the urinary tract, with Escherichia coli being the most commonly isolated pathogen and age being the primary risk factor affecting infection in the early postoperative period (3).

\section{Etiological analysis of early postoperative MDRO infections in SOT recipients}

The perioperative and early postoperative infections in SOT recipients in China are mostly nosocomially acquired MDR Gram-negative bacilli. In addition to Escherichia coli, a member of the family Enterobacteriaceae, the most common pathogens include Acinetobacter baumannii, Pseudomonas aeruginosa, and Stenotrophomonas maltophilia. The isolation rates of Gram-positive cocci are rising annually, with the most common pathogens including Staphylococcus aureus, enterococci, and coagulase-negative staphylococci. Meanwhile, the rate of methicillin resistance has also shown an upward trend (19). Among the 166 patients infected with MDROs early after SOT in our current study, the pathogens were mostly Gram-negative bacteria, accounting for $87 \%$ of all specimens, of which extended spectrum beta-lactamase (ESBL)-producing organisms ranked first, accounting for $66.2 \%$, followed by Acinetobacter baumannii (9.8\%), Pseudomonas aeruginosa (9.3\%), and Klebsiella pneumoniae (8.3\%). In contrast, those infected with Grampositive drug-resistant bacteria accounted for only $10 \%$ among which $S$. aureus was dominant. Drug sensitivity tests showed that carbapenem-resistant (27.1\%), methicillinresistant (6\%), and vancomycin-resistant (1.5\%) strains were predominant, in addition to MDR bacteria (9.2\%) and ultra-broad-spectrum $\beta$-lactam-positive drug-resistant bacteria $(66.2 \%)$. Among them, the 11 iP. aeruginosa strains isolated included 1 pan-drug-resistant $P$. aeruginosa (PDR$\mathrm{PA})$ strain and 10 carbapenem-resistant $P$. aeruginosa strains, while 13 Acinetobacter baumannii strains and 12 Bacillariophyceae strains were resistant to carbapenems. Carbapenems have been considered to be the last line of defense against drug-resistant Gram-negative pathogen infections in the past decade. However, the rapid increase in the detection rates of carbapenem-resistant strains, especially Klebsiella pneumoniae, Pseudomonas aeruginosa, and Acinetobacter baumannii and the gradual evolution of Gramnegative bacteria towards MDR have promoted the more cautious selection of antimicrobials (3).

\section{Complications and outcomes of early postoperative MDRO infections in SOT recipients}

Among the 133 patients with MDRO infections after SOT, urinary tract infections were the most common (43.6\%), followed by pulmonary infections (29.3\%), wound infections (3.8\%) and other infections (33.1\%). These 
infections did not occur definitively at a single site; rather, they could occur at multiple sites simultaneously. Poorly controlled infections could rapidly progress into BSIs, leading to a series of systemic symptoms and even death. In addition to simple infections, there were also other postoperative complications such as acute graft rejection (23.3\%), gastrointestinal bleeding (5.3\%), delayed graft function (DGF) (0.8\%), and intestinal obstruction (0.8\%). Of these patients, $128(96.2 \%)$ were cured, $1(0.8 \%)$ had a serious complication that could be cured, and $4(3 \%)$ died.

Urinary tract infection, a common postoperative infection, may be associated with the use of indwelling urinary catheters. The most frequent type of infection in patients undergoing renal transplantation is urinary tract infection, which is typically caused by Klebsiella pneumoniae and ESBL-producing organisms (20). Simple urinary tract infections have no significant impact on the longterm survival of kidney transplant recipients if managed promptly $(21,22)$. However, poor control of the infection may lead to DGF and even graft damage. Once a systemic infection develops, the risk of death can be high. Most pulmonary infections occurring within 3 months of surgery are hospital-acquired and may be associated with the assisted ventilation in critically ill patients, hypostatic pneumonia due to prolonged immobility, and colonization of drug-resistant bacteria in ICU. The common pathogens include Gram-negative bacilli such as Klebsiella pneumoniae, Escherichia coli, and Pseudomonas aeruginosa; however, Gram-positive pathogens such as Staphylococcus aureus, Streptococcus pneumoniae, and anaerobic bacteria can also be isolated. In a report by Tanaka et al., $92 \%$ of liver transplantation recipients developed bacterial respiratory infections, including at least one episode of pneumonia in $19 \%$ of recipients. Pneumonia after liver transplantation often has a poor prognosis. The incisional infections after renal transplantation are mostly caused by intraincisional hematoma, urinary leakage, or lymph node cysts, with an incidence of $2-47 \%$. The susceptibility factors include the recipient's own physical status, the bacteria and/or fungi carried by the donor, prolonged surgery, delayed postoperative incisional recovery, acute graft rejection, and use of immunosuppressives (23). Once an incisional infection occurs, early diagnosis, effective drainage, and rational use of various drugs (including antibiotics) is important. Adequate low drainage may be performed if necessary.

Acute rejection is common within the first year after a transplant (24). Current research suggests that acute rejection is mainly related to insufficient doses of immunosuppressants, autoimmune diseases in recipients, young age of the recipient, HLA-DR mismatch, long cold ischemia time, and old age of the donor. Once an acute rejection occurs, it can be treated with high-dose pulse intravenous steroids. Gastrointestinal bleeding may be related to surgical stress and the use of high-dose glucocorticoids, while intestinal obstruction is considered a complication of general anesthesia.

With the development of technology, immunosuppressive agents can greatly improve the survival rate of grafts and reduce rejection reactions. However, due to the side effects of immunosuppressants, the incidence of various infections in patients after surgery is very high, especially the infection of multi-drug resistant bacteria. Sometimes due to the lack of effective antibiotics, it can be severely life-threatening. So Infection-related symptoms and laboratory indicators, and the blood concentration of immunosuppressants should be closely monitored. When serious life-threatening infections occur, the Immunosuppressants should be reduced or stopped.

\section{Limitations and prospects}

The results of our current study highlighted the challenges of MDRO infections after SOT. However, our study was limited by its single-center design and failed to follow up patients for longer-term complications and their treatment patterns, which need to be further explored in future clinical studies with larger sample sizes and longer observation periods. As the pathogens and sites of infection of postoperative MDRO infections vary in different time and regions, regular analysis of the clinical features of postoperative MDRO infections in SOT recipients will be valuable for healthcare professionals and their patients.

\section{Acknowledgments}

Funding: None.

\section{Footnote}

Reporting Checklist: The authors have completed the STROBE reporting checklist. Available at http://dx.doi. org/10.21037/apm-21-777

Data Sharing Statement: Available at http://dx.doi. org/10.21037/apm-21-777 
Conflicts of Interest: All authors have completed the ICMJE uniform disclosure form (available at http://dx.doi. org/10.21037/apm-21-777). The authors have no conflicts of interest to declare.

Ethical Statement: The authors are accountable for all aspects of the work in ensuring that questions related to the accuracy or integrity of any part of the work are appropriately investigated and resolved. This study was conformed to the provisions of the Declaration of Helsinki and was approved by the Clinical Research and Application Ethics Committee of the Second Affiliated Hospital of Guangzhou Medical University (No. 2018-QCC-ks-01). Written informed consent was obtained from the patient.

Open Access Statement: This is an Open Access article distributed in accordance with the Creative Commons Attribution-NonCommercial-NoDerivs 4.0 International License (CC BY-NC-ND 4.0), which permits the noncommercial replication and distribution of the article with the strict proviso that no changes or edits are made and the original work is properly cited (including links to both the formal publication through the relevant DOI and the license). See: https://creativecommons.org/licenses/by-nc-nd/4.0/.

\section{References}

1. Silva JT, Fernández-Ruiz M, Aguado JM. Multidrugresistant Gram-negative infection in solid organ transplant recipients: implications for outcome and treatment. Curr Opin Infect Dis 2018;31:499-505.

2. Kritikos A, Manuel O. Bloodstream infections after solidorgan transplantation. Virulence 2016;7:329-40.

3. Wilmes D, Coche E, Rodriguez-Villalobos $\mathrm{H}$, et al. Bacterial pneumonia in kidney transplant recipients. Respir Med 2018;137:89-94.

4. Cinar G, Kalkan İA, Azap A, et al. CarbapenemaseProducing Bacterial Infections in Patients With Liver Transplant. Transplant Proc 2019;51:2461-5.

5. Silva JT, Fernández-Ruiz M, Aguado JM, et al. Multidrugresistant Gram-negative infection in solid organ transplant recipients: implications for outcome and treatment. Curr Opin Infect Dis 2018;31:499-505.

6. Diaz-Nieto R, Lykoudis PM, Davidson BR. Recipient body mass index and infectious complications following liver transplantation. HPB (Oxford) 2019;21:1032-8.

7. Gagliotti C, Morsillo F, Moro ML, et al. Infections in liver and lung transplant recipients: a national prospective cohort. Eur J Clin Microbiol Infect Dis 2018;37:399-407.

8. Bahrami A, Shams SF, Eidgahi ES, et al. Epidemiology of Infectious Complications in Renal Allograft Recipients in the First Year After Transplant. Exp Clin Transplant 2017;15:631-5.

9. Sato A, Kaido T, Iida T, et al. Bundled strategies against infection after liver transplantation: Lessons from multidrug-resistant Pseudomonas aeruginosa. Liver Transpl 2016;22:436-45.

10. Park J, Kim BW, Choi HJ, et al. Risk stratification for early bacteremia after living donor liver transplantation: a retrospective observational cohort study. BMC Surg 2020;20:2.

11. Zhong ZQ, Luo AJ, Wan QQ, et al. Pseudomonas Aeruginosa Infection Among Liver Transplant Recipients: A Clinical Analysis of 15 Cases. Transplant Proc 2016;48:2130-4.

12. Ye QF, Zhou W, Wan QQ. Donor-derived infections among Chinese donation after cardiac death liver recipients. World J Gastroenterol 2017;23:5809-16.

13. Sullivan T, Weinberg A, Rana M, et al. The Epidemiology and Clinical Features of Clostridium difficile Infection in Liver Transplant Recipients. Transplantation 2016;100:1939-43.

14. Lewis JD, Barros AJ, Sifri CD. Comparison of risk factors and outcomes of daptomycin-susceptible and -nonsusceptible vancomycin-resistant Enterococcus faecium infections in liver transplant recipients. Transpl Infect Dis 2018;20:e12856.

15. Feijó MS, Galdino-Vasconcelos MR, Simões V, et al. Impact of Donor Positive Blood Culture in Deceased Donor Liver Transplantation. Transplant Proc 2020;52:1236-42.

16. Errico G, Gagliotti C, Monaco M, et al. Colonization and infection due to carbapenemase-producing Enterobacteriaceae in liver and lung transplant recipients and donor-derived transmission: a prospective cohort study conducted in Italy. Clin Microbiol Infect 2019;25:203-9.

17. Takemura Y, Hibi T, Shinoda M, et al. Methicillinresistant Staphylococcus aureus carriers are vulnerable to bloodstream infection after living donor liver transplantation. Clin Transplant 2019;33:e13753.

18. Dohna Schwake C, Guiddir T, Cuzon G, et al. Bacterial infections in children after liver transplantation: A single-center surveillance study of 345 consecutive transplantations. Transpl Infect Dis 2020;22:e13208.

19. Yu YT, Ma PL. Prevention and control of multidrugresistant organisms:guidelines vs practice. Zhonghua Yi 
Xue Za Zhi 2019;99:1945-8.

20. Gołębiewska JE, Krawczyk B, Wysocka M, et al. Host and pathogen factors in Klebsiella pneumoniae upper urinary tract infections in renal transplant patients. J Med Microbiol 2019;68:382-94.

21. Rodrigues Dos Santos BG, Amaral ES Jr, Fernandes PFCBC, et al. Urinary Tract Infections and Surgical Site Infections due to Carbapenem-Resistant Enterobacteriaceae in Renal Transplant. Transplant Proc 2016;48:2050-5.

22. Illesy L, Szabo-Pap M, Toth F, et al. Bacterial Infections After Kidney Transplantation: A Single-Center

Cite this article as: Jin M, Zeng L, Zhang W, Deng X, Li J, Zhang W. Clinical features of multidrug-resistant organism infections in early postoperative solid organ transplantation in a single center. Ann Palliat Med 2021;10(4):4555-4562. doi: 10.21037/apm-21-777
Experience. Transplant Proc 2016;48:2540-3.

23. Tanaka S, Geneve C, Tebano G, et al. Morbidity and mortality related to pneumonia and TRACHEOBRONCHITIS in ICU after lung transplantation. BMC Pulm Med 2018;18:43.

24. Hu R, Barratt DT, Coller JK, et al. No Major Effect of Innate Immune Genetics on Acute Kidney Rejection in the First 2 Weeks Post-Transplantation. Front Pharmacol 2020;10:1686.

(English Language Editor: J. Gray) 